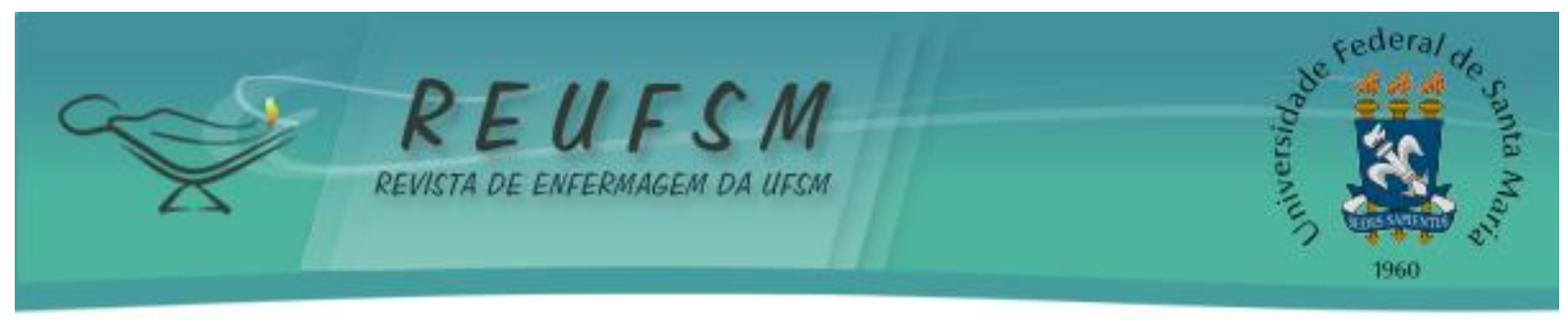

ARTIGO ORIGINAL

\title{
CONHECIMENTO DE ESTUDANTES ADOLESCENTES ACERCA DO HIVIAIDS
}

KNOWLEDGE OF ADOLESCENT STUDENTS ABOUT HIVIAIDS

\section{CONOCIMIENTOS DE ESTUDIANTES ADOLESCENTES SOBRE VIHISIDA}

Rebeca Coelho de Moura Angelim ${ }^{1}$
Fátima Maria da Silva Abrão
Luciana da Rocha Cabral
Síngara Borba de Araújo Queiroz
Rafaela Marrise do Monte Freitas
Mirian Domingos Cardoso

Doi: $10.5902 / 2179769214869$

RESUMO: Objetivo: verificar o conhecimento de estudantes adolescentes sobre o HIV/AIDS. Método: estudo exploratório-descritivo com abordagem quantitativa, realizado com 298 estudantes de duas escolas públicas, em Recife, Pernambuco, Brasil. Para coleta de dados utilizou-se um questionário autoaplicável. Resultados: o estudo aponta que a maioria dos adolescentes já deu início a vida sexual $(53,4 \%)$; no que diz respeito os métodos preventivos, $44,1 \%$ relataram já ter utilizado, sendo o preservativo o mais frequente. A maioria (92\%) declarou saber as formas de transmissão do HIV/AIDS. A televisão $(75,2 \%)$ foi a principal fonte de informação sobre o HIV/AIDS. Conclusão: os adolescentes apesar de terem conhecimento sobre o modo de transmissão do HIV/AIDS, não quer dizer que realizam práticas sexuais seguras, dessa forma, urge a necessidade de implementar políticas de promoção e proteção da saúde no ambiente escolar.

Descritores: Adolescente; Síndrome de imunodeficiência adquirida; HIV; Conhecimento.

ABSTRACT: Aim: to verify the knowledge of adolescent students about HIVIAIDS. Method: exploratory descriptive study with quantitative approach, conducted with 298 students from two public schools of Recife, Pernambuco, Brazil. A self-administered questionnaire was used for data collection. Results: the study points out that most teenagers already initiated sex life (53.4\%); regarding preventive methods, $44.1 \%$ reported having used them, condoms being the most frequent. Most students (92\%) reported knowing the HIVIAIDS transmission routes. Television was the main source of information about HIVIAIDS. Conclusion: despite having knowledge about HIV/AIDS transmission, that doesn't mean that the adolescents perform safe sexual practices, thus, there is a need to implement policies of health promotion and protection in the school environment.

Descriptors: Adolescent; Acquired immunodeficiency syndrome; HIV; Knowledge.

\footnotetext{
1 Enfermeira. Mestranda do Programa Associado de Pós-Graduação em Enfermagem UPE/UEPB. Recife, PE, Brasil. E-mail: rebecaangelim@hotmail.com

${ }^{2}$ Enfermeira. Doutora em Enfermagem. Programa Associado de Pós-Graduação em Enfermagem UPE/UEPB. Recife, PE, Brasil. E-mail: abraofatima@gmail.com

${ }^{3}$ Graduanda do curso de Enfermagem pela Universidade de Pernambuco/Faculdade de Enfermagem Nossa Senhora das Graças (UPE/FENSG). Recife, PE, Brasil. E-mail: lucabral06@hotmail.com

${ }^{4}$ Enfermeira. Graduada pela Universidade de Pernambuco/Faculdade de Enfermagem Nossa Senhora das Graças (UPE/FENSG). Recife, PE, Brasil. E-mail: singara_borba@hotmail.com

${ }^{5}$ Enfermeira. Graduada pela Universidade de Pernambuco/Faculdade de Enfermagem Nossa Senhora das Graças (UPE/FENSG). Recife, PE, Brasil. E-mail: rafamarri@hotmail.com

${ }^{6}$ Enfermeira. Doutora em Epidemiologia. Faculdade de Enfermagem Nossa Senhora das Graças (FENSG/UPE). Recife, PE, Brasil. E-mail: mirian.domingos@upe.br
} 


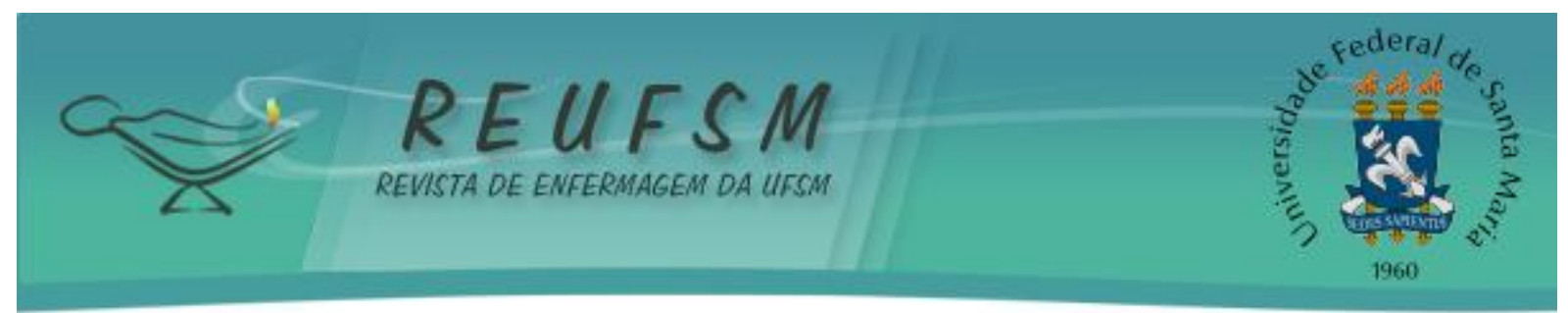

RESUMEN: Objetivo: verificar el conocimiento de los estudiantes adolescentes sobre VIH/SIDA. Método: estudio exploratorio-descriptivo, cuantitativo, realizado con 298 estudiantes de dos escuelas públicas de Recife, Pernambuco, Brasil. Fue utilizado cuestionario auto administrado para recopilar los datos. Resultados: el estudio muestra que la mayoría de los adolescentes ya iniciaron su vida sexual $(53,4 \%)$; con respecto a los métodos de prevención, 44,1\% declaró haber utilizado alguna vez, siendo el más frecuente el preservativo. La mayoría (92\%) informó conocer las formas de transmisión del VIH/SIDA. La televisión fue la principal fuente de información sobre VIH/SIDA. Conclusión: a pesar de los adolescentes poseer conocimiento sobre el modo de transmisión del VIH/SIDA, no significa que realicen prácticas sexuales seguras, así, es necesario implementar políticas de promoción y protección de la salud en el ambiente escolar.

Descriptores: Adolescente; Síndrome de inmunodeficiencia adquirida; VIH; Conocimiento.

\section{INTRODUÇÃO}

A adolescência é um período de transição entre a infância e a fase adulta, compreendida, segundo a Organização Mundial de Saúde (OMS), por indivíduos com faixa etária de 10 a 19 anos, caracterizada pelo desenvolvimento da puberdade, mudanças fisiológicas e corporais, ampliação dos aspectos emocionais e descoberta da sexualidade. ${ }^{1}$ Neste sentido, torna-se necessário realizar abordagens acerca do comportamento e atitude sexual dos adolescente, e consequentemente o nível de instrução destes quanto às Doenças Sexualmente Transmissíveis (DST), principalmente o HIV/AIDS, em virtude, por exemplo, da prática sexual desprotegida, iniciação sexual precoce, múltiplos parceiros e uso de drogas injetáveis.

No Brasil, no período de 1980 a 2013, segundo dados do boletim epidemiológico, 15.480 casos de AIDS foram notificados no Sistema de Informação de Agravos de Notificação na população com idade entre 10 e 19 anos. ${ }^{2}$ Desse total, 8.007 foram identificados entre jovens do sexo masculino, e 7.464, do sexo feminino. ${ }^{2}$ Por meio desses dados, é notório visualizar que diante do potencial epidêmico e expansão, a AIDS ainda é um problema de saúde pública, a nível global, e cada vez mais prevalente em adolescentes.

Vale ressaltar que é essencial disseminar informações sobre a utilização do preservativo, visto que é o método mais eficaz de prevenção da AIDS e de outras DST ${ }^{3}$, estando acessível a toda a população brasileira de forma gratuita. Assim, a adoção de medidas educativas com o intuito de reduzir os riscos aos quais os adolescentes estão expostos, deve ser priorizado pelos governantes por meio de programas focados em políticas públicas direcionadas a esses indivíduos, com ênfase na prevenção e promoção da saúde.

No âmbito do ensino, cabe à escola estimular a discussão e a implementação de estratégias que priorizem a prática sexual segura no espaço de convivência dos adolescentes ${ }^{4}$, uma vez que é nesse ambiente em que os mesmos podem atuar como seres ativos na construção do seu próprio conhecimento. Merece destacar ainda a importância da família em dialogar com a escola, tornando o processo de educação sexual emancipatório, visto que a participação da família pode colaborar para a eficácia das intervenções, através do desenvolvimento de diálogos abertos para o esclarecimento dos adolescentes e comunidade em geral. ${ }^{5}$

Outrossim, os serviços de Atenção Básica por meio do Programa Saúde na Escola, necessitam atuar no ambiente escolar de modo a inserir esse grupo etário nas suas práticas assistenciais, atentando para as necessidades de saúde dessa clientela. Para tal, é fundamental a realização de um atendimento individualizado, de forma privativa e confidencial, acerca da saúde sexual e reprodutiva para os adolescentes, visando reduzir os riscos de possíveis infecções e futuras transmissões das DST, em especial, o HIV/AIDS. ${ }^{6}$ 


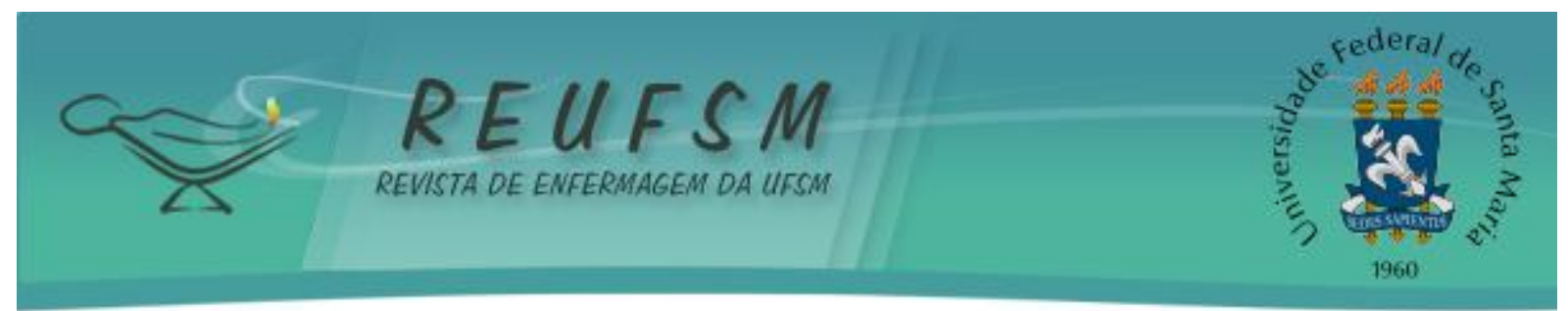

É inegável a necessidade de desenvolver estudos que busquem investigar os conhecimentos de adolescentes escolares acerca das DST, em particular, o HIV/AIDS, em detrimento da maior exposição e da crescente incidência de acometidos pela mencionada patologia. Além disso, trabalhar os conhecimentos desses indivíduos no ambiente escolar torna-se propício, tendo em vista que é um local em que circulam e costumam compartilhar informações e experiências nos mais diversos temas que circunda essa faixa etária, principalmente quando se trata de assuntos relacionados à sexualidade, além do que, é nesta fase que se exacerbam os hormônios masculinos e femininos, estimulando a realização do ato sexual.

Diante das considerações apresentadas, o presente estudo teve o objetivo de verificar o conhecimento de estudantes adolescentes sobre o HIV/AIDS.

\section{MÉTODO}

Trata-se de uma pesquisa exploratório-descritiva com abordagem quantitativa. A amostra do estudo foi constituída por 298 adolescentes de duas escolas públicas, da cidade de Recife, Pernambuco, Brasil. Como critérios de inclusão foram selecionados alunos regularmente matriculados no $9^{\circ}$ Ano do Ensino Fundamental II e no Ensino Médio que tivessem idade entre 13 e 19 anos, e que estivesse com o Termo de Consentimento Livre e Esclarecido (TCLE) assinado pelos indivíduos com idade a partir de 18 anos, e no caso de indivíduos com idade entre 13 e 17 anos, o TCLE foi assinado pelos pais ou responsável legal do menor.

Os dados foram coletados no período de outubro a dezembro de 2012, por meio de um questionário autoaplicável composto por questões pertinentes ao objetivo da pesquisa, contendo variáveis de caracterização dos participantes (idade, sexo, orientação sexual e escolaridade), investigação dos conhecimentos sobre HIV/AIDS (formas de transmissão, cura e suspeita de contaminação), início da atividade sexual, dos métodos preventivos, das fontes de informação sobre o HIV/AIDS e os tipos de DST que os indivíduos conheciam. Os estudantes responderam ao questionário no ambiente de sala de aula das instituições de ensino selecionadas para o estudo.

Os achados obtidos pela investigação foram digitados no programa EpiData 3.1. 0 programa estatístico utilizado para a obtenção dos cálculos estatísticos foi o Statistical Package for the Social Sciences (SPSS) na versão 17.

Para a análise quantitativa, foram utilizadas distribuições absolutas e percentuais, e técnicas de estatística inferencial através do teste Qui-quadrado de Pearson, quando não houve condições para utilizá-lo. A margem de erro utilizada nas decisões dos testes estatísticos foi de $5 \%$.

No tocante aos aspectos éticos, o estudo foi desenvolvido em consonância com a Resolução $196 / 1996^{7}$, do Conselho Nacional de Saúde, na época do estudo, atualizada pela Resolução 466/2012, que regulamenta a pesquisa com seres humanos no país. O projeto de pesquisa foi aprovado pelo Comitê de Ética em Pesquisa da Universidade de Pernambuco (UPE), sob Certificado de Apresentação para Apreciação Ética (CAAE) de $n^{\circ}$ 03618912.5.0000.5207.

\section{RESULTADOS}

Quanto à caracterização dos participantes do estudo, a tabela 1, a seguir, evidenciou que a idade dos adolescentes variou de 13 a 19 anos, e o sexo feminino foi o mais prevalente. No que diz respeito à escolaridade, a maioria dos participantes cursava a $1^{\text {a }}$ série do ensino médio (Tabela 1 ). 


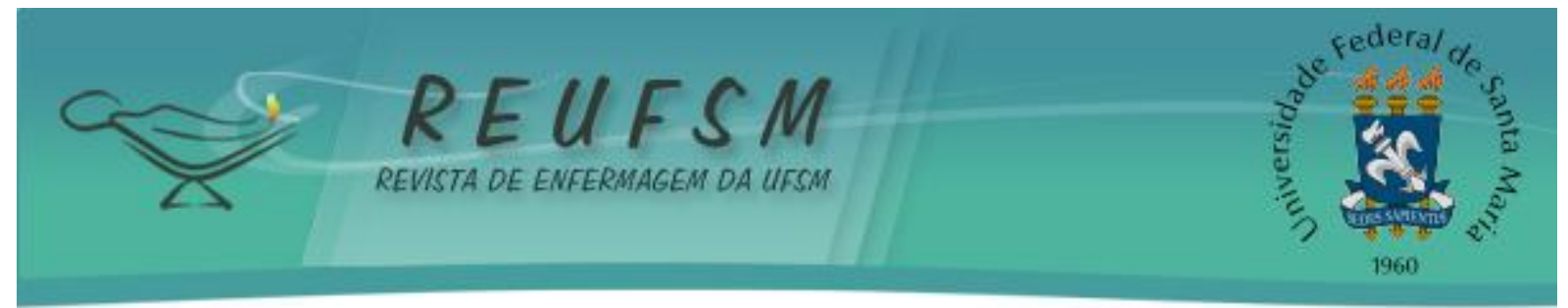

Tabela 1 - Distribuição segundo a caracterização dos adolescentes participantes do estudo. Recife (PE), Brasil. 2012

\begin{tabular}{|c|c|}
\hline Variável & $\mathrm{n}(\%)$ \\
\hline Total & $298(100)$ \\
\hline \multicolumn{2}{|l|}{ Faixa etária } \\
\hline 13 a 16 & $155(52,0)$ \\
\hline 17 a 19 & $143(48,0)$ \\
\hline \multicolumn{2}{|l|}{ Sexo } \\
\hline Masculino & $127(42,6)$ \\
\hline Feminino & $171(57,4)$ \\
\hline \multicolumn{2}{|l|}{ Orientação sexual } \\
\hline Heterossexual & $265(89)$ \\
\hline Homossexual & $10(3,3)$ \\
\hline Bissexual & $5(1,7)$ \\
\hline Outro & $10(3,3)$ \\
\hline Não informado & $8(2,7)$ \\
\hline \multicolumn{2}{|l|}{ Escolaridade } \\
\hline $9^{a}$ série & $64(21,5)$ \\
\hline 1 a série do ensino médio & $96(32,2)$ \\
\hline $2^{2}$ série do ensino médio & $70(23,5)$ \\
\hline $3^{\mathrm{a}}$ série do ensino médio & $68(22,8)$ \\
\hline
\end{tabular}

No tocante a atividade sexual, 53,4\% dos 293 que responderam a pergunta referiu já ter iniciado a prática sexual, demonstrando significância ao correlacionar com o sexo, havendo maior incidência nos indivíduos do sexo masculino. Desses, 82,7\% utilizaram, em algum momento, um método preventivo, com destaque para a camisinha (Tabela 2 ).

Tabela 2 - Distribuição da frequência e percentual dos adolescentes do estudo relacionados à relação sexual e prevenção, segundo o sexo. Recife (PE), Brasil. 2012

\begin{tabular}{|c|c|c|c|c|c|}
\hline \multirow{3}{*}{ Variável } & \multicolumn{4}{|c|}{ Sexo } & \multirow{3}{*}{ Valor de $\mathrm{p}$} \\
\hline & \multicolumn{2}{|c|}{ Masculino } & \multicolumn{2}{|c|}{ Feminino } & \\
\hline & $\mathrm{n}$ & $\%$ & $\mathrm{~N}$ & $\%$ & \\
\hline \multicolumn{6}{|c|}{ - Já teve relacão sexual? } \\
\hline Sim & 86 & 70,0 & 71 & 41,7 & $\mathrm{p}^{(1)}<0,001^{*}$ \\
\hline Não & 37 & 30,0 & 99 & 58,3 & \\
\hline TOTAL & 123 & 100,0 & 170 & 100,0 & \\
\hline \multicolumn{6}{|c|}{$\begin{array}{l}\text { - Se sim, utilizou algum método } \\
\text { preventivo? }\end{array}$} \\
\hline Sim & 70 & 81,4 & 60 & 84,5 & $\mathrm{p}^{(1)}=0,747$ \\
\hline Não & 16 & 18,6 & 11 & 15,5 & \\
\hline TOTAL & 86 & 100,0 & 71 & 100,0 & \\
\hline
\end{tabular}

Nota $1\left(^{*}\right)$ : Diferença significativa ao nível de $5,0 \%$.

Nota 2 (1): Através do teste Qui-quadrado de Pearson.

Quanto à fonte para a obtenção de informação sobre HIV, a televisão foi citada pela maioria dos sujeitos do estudo como a principal fonte, e a escola apareceu em segundo lugar (Tabela 3). Pode-se observar ainda, que a escola foi a única fonte de informação que apresentou uma considerável significância ao relacionar com a idade dos adolescentes. 


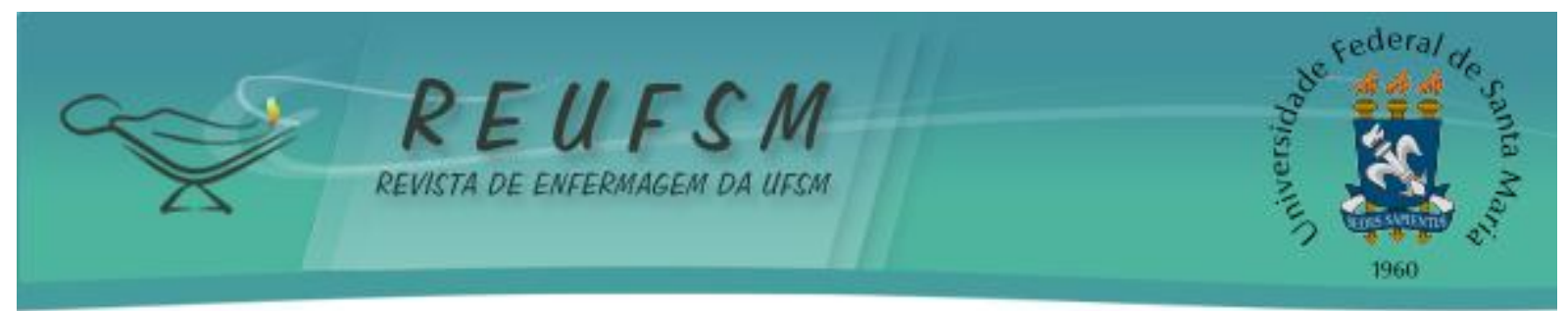

Tabela 3 - Distribuição dos dados relacionados às fontes de informação acerca do HIV/AIDS segundo a faixa etária. Recife (PE), Brasil. 2012.

\begin{tabular}{|c|c|c|c|c|c|}
\hline \multirow[t]{2}{*}{ Variável } & \multicolumn{2}{|c|}{13 a 16} & \multicolumn{2}{|c|}{17 a 19} & \multirow{2}{*}{ Valor de $\mathrm{p}$} \\
\hline & $\mathrm{n}$ & $\%$ & $\mathrm{n}$ & $\%$ & \\
\hline \multicolumn{6}{|l|}{ Internet } \\
\hline Sim & 73 & 47,1 & 73 & 51,0 & \multirow[t]{2}{*}{$\mathrm{p}^{(1)}=0,495$} \\
\hline Não & 82 & 52,9 & 70 & 49,0 & \\
\hline \multicolumn{6}{|l|}{ Jornal } \\
\hline Sim & 51 & 32,9 & 47 & 32,9 & \multirow[t]{2}{*}{$\mathrm{p}^{(1)}=0,994$} \\
\hline Não & 104 & 67,1 & 96 & 67,1 & \\
\hline \multicolumn{6}{|l|}{ Revista } \\
\hline Sim & 29 & 18,7 & 37 & 25,9 & \multirow[t]{2}{*}{$\mathrm{p}^{(1)}=0,137$} \\
\hline Não & 126 & 81,3 & 106 & 74,1 & \\
\hline \multicolumn{6}{|c|}{ Televisão } \\
\hline Sim & 113 & 72,9 & 111 & 77,6 & \multirow[t]{2}{*}{$\mathrm{p}^{(1)}=0,346$} \\
\hline Não & 42 & 27,1 & 32 & 22,4 & \\
\hline \multicolumn{6}{|l|}{ Casa } \\
\hline $\mathrm{Sim}$ & 57 & 36,8 & 51 & 35,7 & \multirow[t]{2}{*}{$\mathrm{p}^{(1)}=0,842$} \\
\hline Não & 98 & 63,2 & 92 & 64,3 & \\
\hline \multicolumn{6}{|l|}{ Escola } \\
\hline Sim & 85 & 54,8 & 96 & 67,1 & \multirow[t]{2}{*}{$p^{(1)}=0,030^{*}$} \\
\hline Não & 70 & 45,2 & 47 & 32,9 & \\
\hline \multicolumn{6}{|l|}{ USF } \\
\hline Sim & 25 & 16,1 & 25 & 17,5 & \multirow[t]{3}{*}{$\mathrm{p}^{(1)}=0,755$} \\
\hline Não & 130 & 83,9 & 118 & 82,5 & \\
\hline TOTAL & 155 & 100,0 & 143 & 100,0 & \\
\hline
\end{tabular}

Nota $1\left(^{*}\right)$ : Diferença significativa ao nível de 5,0\%.

Nota 2 (1): Através do teste Qui-quadrado de Pearson.

Nota: Os participantes marcaram mais de uma opção.

Conforme mostra a Tabela 4, em relação aos conhecimentos sobre como se transmite o HIV, a maioria respondeu ser através de relação sexual ou ao compartilhar seringas. Sobre a cura da AIDS, 214 afirmaram que não tem cura, sendo assim esses dados demonstram que os adolescentes possuem relativo conhecimento acerca do HIV/AIDS, tendo em vista que é um tema frequentemente abordado pela sociedade. 


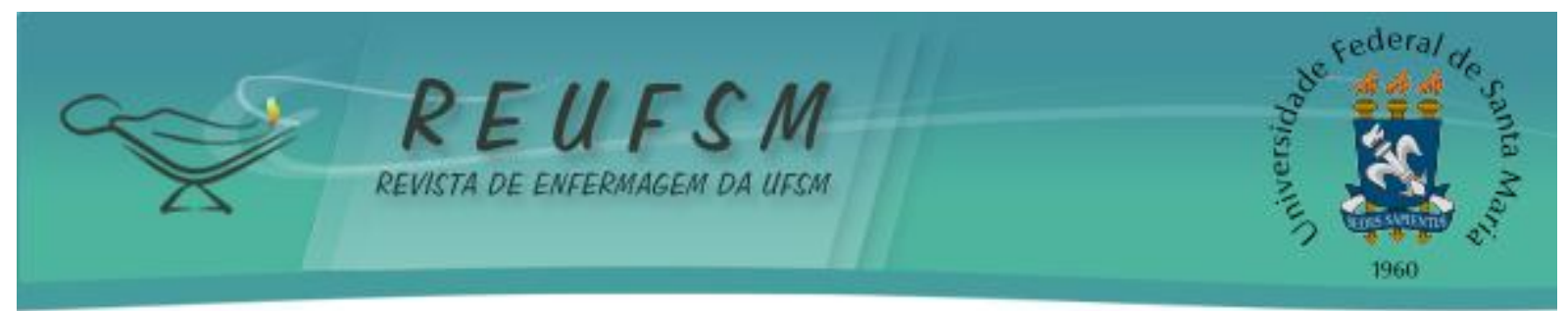

Tabela 4 - Distribuição dos participantes segundo os dados relacionados aos conhecimentos quanto a transmissão, cura e contaminação. Recife (PE), Brasil. 2012

\begin{tabular}{lcc}
\hline Variável & $\mathrm{N}$ & $\%$ \\
\hline TOTAL & 298 & 100,0 \\
- Sabe como se transmite HIV? & 16 & 5,4 \\
Beijo na boca & 1 & 0,3 \\
Picada de inseto & 261 & 87,6 \\
Relação sexual ou compartilhar seringas & 6 & 2,0 \\
Não sabe & 14 & 4,7 \\
Não informado & & \\
- AlDS tem cura? & 18 & 6,04 \\
Sim & 214 & 71,81 \\
Não & 48 & 16,11 \\
Não sabe & 18 & 6,04 \\
Não informado & & 2,7 \\
- Já suspeitou está contaminado pelo HIV? & 8 \\
Sim & 266 \\
Não & 7 & 89,3 \\
Não sabe & 17 & 2,3 \\
Não informado & 5,7 \\
\hline
\end{tabular}

Em relação à contaminação (Tabela 4), oito já suspeitaram estar infectado pelo vírus em algum momento, desses, apenas três procuraram alguma orientação quanto às medidas cabíveis a serem tomadas e, por conseguinte esclarecimentos sobre esta temática.

Por fim, foram investigadas as DST conhecidas pelos adolescentes. Sendo assim, constatou-se que $72,2 \%$ dos adolescentes mencionaram pelo menos uma, sendo elas, o HIV, AIDS, Sífilis ou Cancro duro, Gonorreia, Herpes, Cancro Mole, Hepatite, Condiloma acuminado ou HPV ou Crista de Galo e Mula (Linfogranuloma Venéreo). Enquanto que $15,1 \%$ não informaram e $12,7 \%$ não souberam.

\section{DISCUSSÃO}

Este estudo foi caracterizado por se tratar de adolescentes de 13 a 19 anos com predominância do sexo feminino. No que tange os conhecimentos gerais acerca das DST, pode-se observar que não é um tema desconhecido pelos adolescentes desta pesquisa.

A pesquisa apontou que $53,4 \%$ dos adolescentes afirmaram já ter tido relação sexual, em se tratando dos adolescentes do sexo masculino, observa-se tendência a predisposição da iniciação sexual mais precocemente. Porém, merece destacar que o uso do preservativo e sua importância mostraram equivalência ao correlacionar com o gênero, tendo em vista que $82,7 \%$ dos adolescentes em geral disseram ter utilizado alguma vez um método de prevenção.

A iniciação precoce de atividade sexual revelada neste estudo aponta para a necessidade de incorporar práticas educativas direcionadas para promoção e manutenção da saúde desse grupo etário com o intuito de prevenir o adoecimento dessa população por uma DST. ${ }^{8}$ Sendo assim, pode-se destacar a utilização de oficinas em grupo sobre sexualidade como um procedimento pedagógico e estratégico para ofertar esclarecimentos de dúvidas e questionamentos sobre os mais diversos temas que englobam a saúde sexual do adolescente. ${ }^{5}$

No que diz respeito às fontes de informação que os adolescentes utilizam para adquirir conhecimento sobre as DST, a televisão $(75,2 \%)$ apresentou-se como a mais mencionada pelos estudantes a respeito do tema HIV/AIDS, seguida pela escola e pela internet. Nessa perspectiva, outro estudo ${ }^{8}$ também obteve a mídia televisiva como primeira opção de mecanismo de informação sobre o assunto, com 91,6\%. Tal achado 


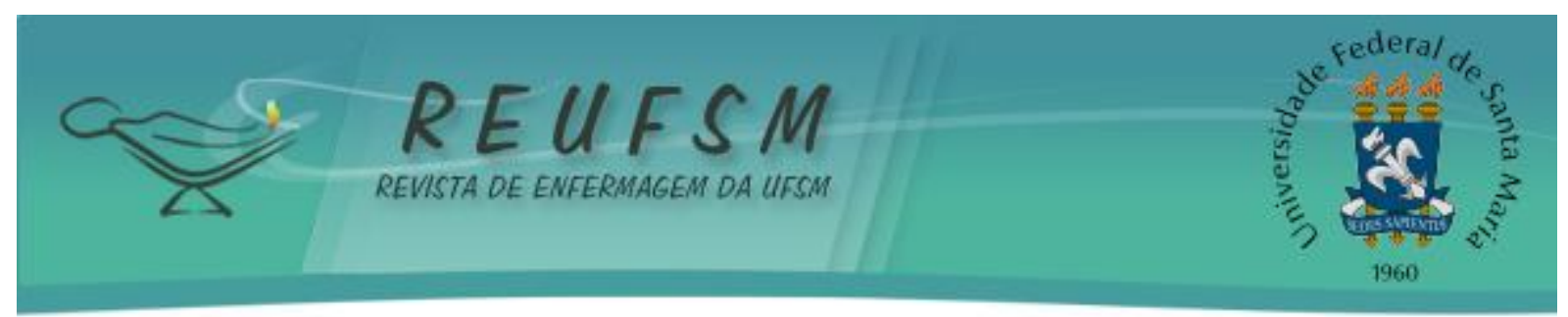

revela o poder desse veículo na comunidade juvenil e desperta a possibilidade de ausência ou pouco diálogo sobre esse tema em discussões familiares. ${ }^{9}$

A televisão é um dos meios de comunicação mais utilizados pela maioria das pessoas e de grande influência no cotidiano delas, porém são poucos os programas educacionais que as redes de televisão oferecem abordando a temática em questão. Observa-se, ainda, que as informações encontradas sobre AIDS e sexualidade estão preferencialmente voltadas para o uso do preservativo nas relações sexuais. Portanto, é importante mencionar que é preciso investir em programas de políticas públicas que envolvam essa temática, para que a população tenha mais conhecimento.

Merece destacar ainda a escola, citada em segundo lugar $(60,7 \%)$ como a fonte de informação mais acessada, ambiente que representa um papel fundamental na vivência dos estudantes, promotor do processo ensino-aprendizagem e, por isso, configura-se como um local favorável para as discussões e questionamentos sobre os mais diversos assuntos com os professores e os educadores.

Assim, considerando que a escola é um lugar onde os adolescentes passam a maior parte do seu tempo e local de destaque na formação e na socialização, devem-se desenvolver trabalhos que abordem a sexualidade humana e sua evolução, o que resultará na construção de conhecimentos e habilidades nas mais diversificadas áreas do saber. Além disso, é fonte de apoio social e afetivo aos estudantes e pode garantir intervenções importantes no desenvolvimento de práticas protetoras da saúde. ${ }^{4,10}$

Nesse sentido, programas educacionais são indispensáveis no sistema de ensino público, com o objetivo de enfatizar as consequências do sexo desprotegido como prática de risco para se adquirir alguma DST. No entanto, a escola, por vezes, apresenta dificuldades em executar sua função social e pedagógica frente à temática, pois, para o desenvolvimento das ações promotoras desse conhecimento, é fundamental que a equipe de docentes esteja habilitada e preparada para incluir a discussão no ambiente escolar. ${ }^{11}$

A pesquisa apresentou a internet dentre as três principais fontes de informação sobre o HIV/AIDS (49\%), estando em consonância com estudo realizado com estudantes de escola pública que teve como resultado internet e amigos como preferência dos alunos para obtenção de informações ${ }^{12}$, nesta perspectiva vale ressaltar a presença da informática como meio de aprendizagem presente no cotidiano dos jovens e adultos. ${ }^{5}$

É oportuno destacar que os adolescentes da atualidade assumem características demarcadas pela exposição e pelo estímulo precoce do uso de equipamentos tecnológicos de informação e entretenimento como meio de aprendizagem. Nesse contexto, a internet é apresentada com significados e efeitos que favorecem a liberdade de comunicação, proporcionando formas alternativas para aquisição de conhecimento sobre as temáticas mais diversas. ${ }^{13}$

O estudo apresentou consonância com a literatura atual ao apontar a Unidade Básica de Saúde como a fonte de informação menos mencionada $(16,8 \%) .{ }^{14}$ Isso se deve tanto a pouca oferta quanto a pouca procura. Estudo alerta que é de fundamental importância que, na equipe da Estratégia Saúde da Família (ESF), haja profissionais que consigam integrar e conquistar a confiança desses adolescentes para um adequado ensino da educação em saúde ${ }^{15-}$ ${ }^{16}$, visto que o impacto das ações eficientes realizadas pela ESF voltadas para o público adolescente contribui sobremaneira para prevenir doenças que futuramente poderiam causar prejuízos e conflitos à saúde desse indivíduo e ao meio social que o cerca. ${ }^{15}$

Dessa forma, cabe mencionar que o Programa Saúde na Escola atua como facilitador para realização de estratégias de educação em saúde com vista na prevenção e promoção da saúde, direcionadas aos adolescentes.

Nesse contexto, é pertinente destacar que o enfermeiro, como membro da equipe multiprofissional e educador, exerce função importante na educação dos adolescentes, 


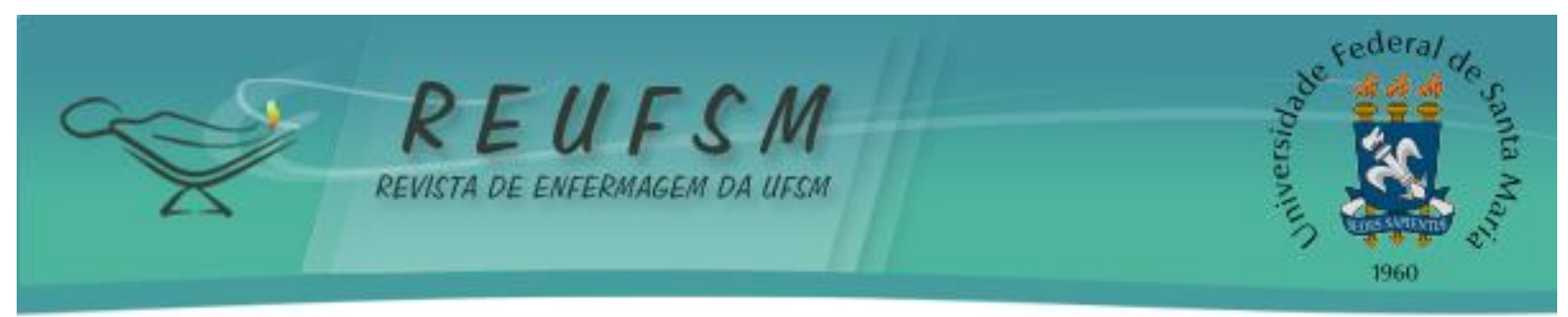

porque proporciona ações de cuidar e estratégias de promoção da saúde de acordo com suas necessidades, com reflexões voltadas para os comportamentos e as práticas em saúde. ${ }^{17}$

Em relação às formas de transmissão, um estudo realizado com estudantes que cursavam o ensino médio de uma rede pública concluiu que $78 \%$ conheciam as formas de infecção do HIV/AIDS. Estudo destaca que, independentemente desses conhecimentos, as práticas sexuais não ocorrem de maneira saudável. ${ }^{12}$ Nessa perspectiva, os dados obtidos pela pesquisa apresentaram-se em conformidade com o estudo citado, porquanto a maioria dos adolescentes (92\%) domina o assunto sobre as formas de transmissão do HIV/AIDS. Entretanto, os dados demonstram a existência de comportamentos que podem predispor à aquisição do vírus, como a não utilização frequente de métodos preventivos durante as relações sexuais.

Estudos mostram que, independentemente do nível socioeconômico, o conhecimento de jovens e adolescentes acerca da transmissão e da prevenção do HIV ainda é limitado, e o uso do preservativo não é frequente. ${ }^{18-19}$ Apenas $33,3 \%$ afirmaram usar o preservativo sempre. ${ }^{19} \mathrm{~A}$ falta do preservativo no momento da relação resulta em práticas sexuais desprotegidas, predispondo os adolescentes à aquisição de alguma DST.

Nessa perspectiva, é relevante fornecer informações, também, aos pais dos adolescentes, para que possam orientar seus filhos a realizarem práticas sexuais saudáveis. ${ }^{18}$ Para isso, são necessárias as intervenções que promovam adequados comportamentos sexuais e ensinamentos do uso correto de métodos contraceptivos e preventivos, assim como a promoção à saúde, com esclarecimentos relacionados às questões de gênero e aos estereótipos acerca do HIV/AIDS. ${ }^{20}$

Considerando que a epidemia de HIV é um dos maiores desafios de saúde pública e que a população adolescente apresenta elevado comportamento de risco, como o baixo nível de conhecimento e a falta de acesso a serviços de prevenção, o uso frequente e correto do preservativo caracteriza-se como a melhor estratégia de prevenção para reduzir a incidência de infecção por meio do HIV. ${ }^{21}$

Tendo em vista as fragilidades e limitações encontradas nos adolescentes inseridos nas escolas contempladas no presente estudo, tornou-se necessário a realização de esclarecimentos sobre o que tange as DST, com ênfase no HIV/AIDS, possibilitando uma abordagem mais abrangente e baseada em conhecimentos científicos. Desse modo, foram explanadas as principais DST, suas formas de transmissão, medidas de prevenção e principais sinais e sintomas, além de esclarecer dúvidas e questionamentos que surgiram entre os participantes.

Nesse contexto, sabendo-se que a saúde sexual e reprodutiva dos adolescentes é um assunto pouco abordado pelos profissionais de saúde, pais/familiares e educadores como um todo, vale ressaltar a necessidade em investir na desmistificação de questões que norteiam a sexualidade de jovens e adolescentes.

\section{CONSIDERAÇÔES FINAIS}

Os achados revelam que o público em estudo possui conhecimento satisfatório acerca dos meios de aquisição do HIV. No entanto, faz necessário fomentar ações de educação em saúde, por meio de oficinas, palestras e políticas públicas direcionadas para esta população, com o intuito de garantir, dentro da realidade local, o conhecimento científico dos adolescentes sobre esse tema, que apresenta grande relevância para a saúde pública brasileira.

Os dados obtidos apresentaram resultados condizentes com a literatura em relação ao não uso frequente do preservativo por adolescentes, apesar de terem ciência que é um dispositivo eficaz para prevenir as DST. Dessa forma, é de grande relevância sensibilizar esses indivíduos sobre a importância do método preventivo, as formas de uso e 


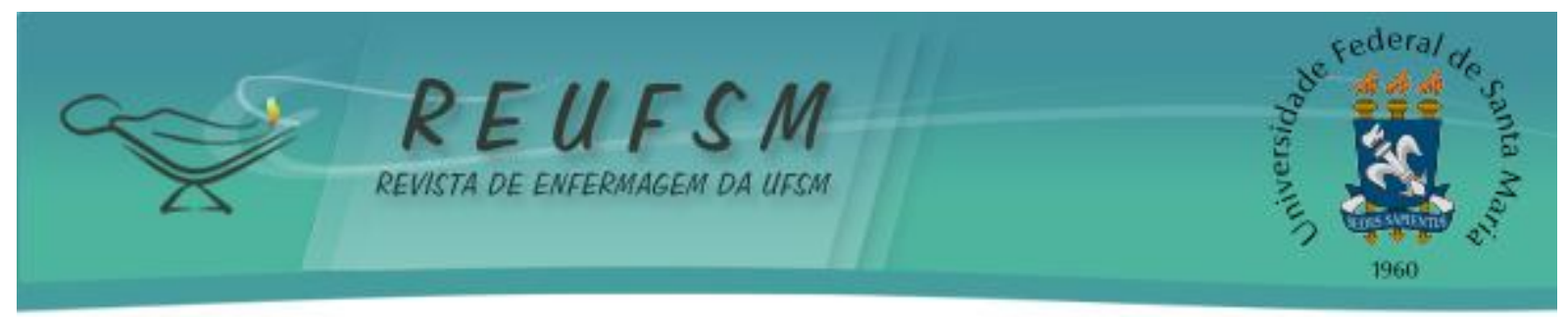

as consequências do não uso, para que possa ser assegurada a adesão do preservativo pelos adolescentes, favorecendo a realização de práticas sexuais saudáveis.

Por fim, este estudo pretende subsidiar novas pesquisas acerca da saúde do adolescente, com a inclusão de participantes com diferentes realidades socioculturais e econômicas, permitindo associar o conhecimento às condições locais dessa população e comportando maiores discussões relacionadas à temática das DST, proporcionando reflexões acerca de práticas sexuais seguras.

Foi possível observar que o presente estudo teve como limitação, o questionário, por ter sido realizado de forma objetiva, carecendo ser com um formato subjetivo para melhor expor a realidade da população estudada.

\section{REFERÊNCIAS}

1. Brasil. Ministério da Saúde. Secretaria de Vigilância em Saúde. Departamento de DST, AIDS e Hepatites Virais. Recomendações para a atenção integral a adolescentes e jovens vivendo com HIV/AIDS. Brasília: Ministério da Saúde; 2013.

2. Brasil. Ministério da Saúde. Secretaria de Vigilância em Saúde. Departamento de DST, AIDS e Hepatites Virais. Boletim Epidemiológico - AIDS e DST. 2013; ano II(1).

3. Eaton LA, Kalichman SC, O'Connell DA, Karchner WD. A strategy for selecting sexual partners believed to pose little/no risks for HIV: serosorting and its implications for HIV transmission. AIDS Care. 2009;21(10):1279-88.

4. Camargo EÁl, Ferrari RAP. Adolescentes: conhecimentos sobre sexualidade antes e após a participação em oficinas de prevenção. Ciênc Saúde Coletiva. 2009;14(3):937-46.

5. Reis VL, Maia ACB. Educação sexual na escola com a participação da família e o uso de novas tecnologias da educação: um levantamento bibliográfico. Cad Educ [Internet]. 2012 [acesso em 2014 mar 15];(41):188-207. Disponível em: http://www.periodicos.ufpel.edu.br/ojs2/index.php/caduc/article/viewFile/2099/1937.

6. Taquette SR, Matos HJ, Rodrigues AO, Bortolotti LR, Amorim E. A epidemia de AIDS em adolescentes de 13 a 19 anos, no município do rio de janeiro: descrição espaço-temporal. Rev Soc Bras Med Trop. 2011;44(4):467-70.

7. Brasil. Ministério da Saúde. Conselho Nacional de Saúde. Resolução CNS n 196, de 10 de outubro de 1996. Diretrizes e normas regulamentadoras de pesquisa envolvendo seres humanos. Brasília (DF): Ministério da Saúde; 1996.

8. Melo MC. Perfil epidemiológico de crianças e adolescentes com SIDA: série histórica de 1985 a 2012. Rev Enferm UFSM [Internet]. 2013 [acesso em 2014 jun 15];3(3):418-28. Disponível em: http://cascavel.ufsm.br/revistas/ojs2.2.2/index.php/reufsm/article/view/8956/pdf.

9. Albuquerque JG, Pinheiro PNC, Lopes MVO, Machado MFAS. Conhecimento deficiente acerca do HIV/AIDS em estudantes adolescentes: identificação de diagnóstico de enfermagem da NANDA. Rev Eletrônica Enferm [Internet]. 2012 [acesso em 2013 abr 30];14(1):104-11. Disponível http: //www.fen.ufg.br/fen_revista/v14/n1/pdf/v14n1a12.pdf.

10. Salganik MJ, Fazito D, Bertoni N, Abdo AH, Mello MB, Bastos Fl. Assessing network scale-up estimates for groups most at risk of HIV/AIDS: evidence from a multiple-method study of heavy drug users in Curitiba, Brazil. Am J Epidemiol. 2011;174(10):1190-6. 


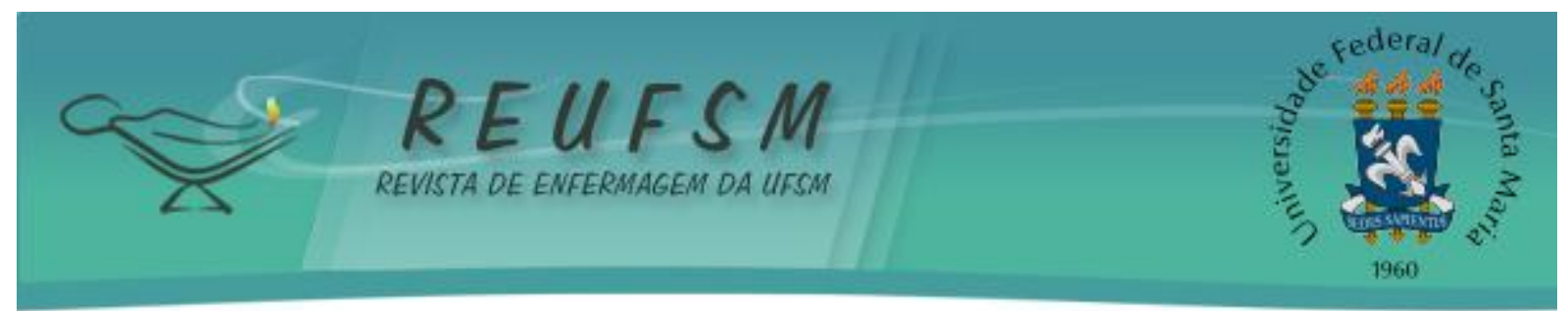

11. Araújo TM, Vieira NFC, Araújo MFM, Pinheiro PNC. Abordagem grupal na prevenção da aids: análise do conhecimento de jovens de Fortaleza. Rev RENE. 2010;11(3):77-85.

12. Pereira MR, Costa RGS. Sexualidade no ensino médio: a escola auxiliando na prevenção da aids. Saúde \& Amb Rev. 2010;5(2):24-30.

13. Spizzirri RCP, Wagner A, Mosmann CP, Armani AB. Adolescência conectada: mapeando o uso da internet em jovens internautas. Psicol Argum. 2012;30(69):327-35.

14. Scoralick GBF, Oliveira OO, Vieira CZ, Santos JN, Nolasco DG. Desconstruindo o mito do início da vida sexual: uma vivência educativa com adolescentes do ensino fundamental de niterói. Rev Pesqui Cuid Fundam [Internet]. 2010 [acesso em 2014 jan 10];2(Supl):558-61. Disponível em: http://www.seer.unirio.br/index.php/cuidadofundamental/article/view/996/pdf_205.

15. Higarashi IH, Baratieri T, Roecker S, Marcon SS. Atuação do Enfermeiro junto aos adolescentes: identificando dificuldades e perspectivas de transformação. Rev Enferm UERJ. 2011;19(3):375-80.

16. Souza TS, Cabral IE, Paula CC. Adolescer de crianças infectadas por transmissão vertical do HIV: Implicações para o cuidado de enfermagem. Rev Enferm UFSM [Internet]. 2011 [acesso em 2014 jun 10];1(3):326-34. Disponível em: http://cascavel.ufsm.br/revistas/ojs-2.2.2/index.php/reufsm/article/view/3188/2381.

17. Ximenes Neto FRG, Aguiar DT, Martins FR, Oliveira EN. Trabalho do enfermeiro na estratégia saúde da família no cuidado ao adolescente - Cariré, Ceará, Brasil. Rev Enferm Prof. 2014;1(1):82-91.

18. Gonçalves H, Gonzalez-Chica DA, Menezes AMB, Hallal PC, Araújo CLP, Dumith SC. Conhecimento sobre a transmissão de HIV/AIDS entre adolescentes com 11 anos de idade do Sul do Brasil. Rev Bras Epidemiol. 2013;16(2):420-31.

19. Bezerra EO, Chaves ACP, Pereira MLD, Melo FRG. Análise da vulnerabilidade sexual de estudantes universitários ao HIV/AIDS. Rev RENE. 2012;13(5):1121-31.

20. Tronco CB, Dell'Aglio DD. Caracterização do comportamento sexual de adolescentes: iniciação sexual e gênero. Gerais: Rev Interinst Psicol [Internet]. 2012 [acesso em 2013 dez 10];5(2):254-69. Disponível em: http://pepsic.bvsalud.org/pdf/gerais/v5n2/v5n2a06.pdf.

21. UNAIDS. Country Progress Report on HIV/AIDS Response, 2012 [Internet]. Ethiopia; 2012 [acesso em 2013 dez 10]. Www.unaids.org/knowyourresponse/countryprogressreports/2012countries

/GAP\%20Report\%202012.pdf.

Data de recebimento: $16 / 07 / 2014$

Data de aceite: 12/03/2015

Contato com autor responsável: Rebeca Coelho de Moura Angelim

Endereço postal: Rua Padre Landim, 292/Apto 504 - Bairro Madalena - CEP 50710-470 Recife/PE/Brasil

E-mail: rebecaangelim@hotmail.com 